\title{
INTEGRAL FIELD SPECTROSCOPY OF IRAS 15206+3342: GAS INFLOWS AND STARBURSTS IN AN ADVANCED MERGER ${ }^{1}$
}

\author{
SAntiago Arribas $^{2,3}$ And Luis Colina 4 \\ Received 2001 November 7; accepted 2002 March 19
}

\begin{abstract}
Integral field optical spectroscopy using the INTEGRAL system (complemented with Hubble Space Telescope WFPC2 imaging) is used to characterize the kinematic and ionization properties of the warm ionized gas in the ultraluminous infrared galaxy IRAS $15206+3342$. These data present kinematic and morphological evidence for a tidal tail connecting the nuclear star-forming regions with a remnant stellar system located at a projected distance of $20 \mathrm{kpc}$ away from the nucleus. The velocity field and the velocity dispersion map indicate the presence of gas flowing inward along the tidal tail, feeding the nuclear regions where the previously detected arc-shaped chain of young stellar clusters is forming stars at a rate of about $150 M_{\odot} \mathrm{yr}^{-1}$. According to theoretical models by Mihos \& Hernquist, these characteristics (e.g., large gas inflows and massive starbursts) are expected during the final coalescence phase of two disk galaxies with bulges. No kinematic evidence for a second nucleus, nor for the presence of young star-forming regions located along the external parts of the tidal tail, is found, further supporting the conclusion that this system is an advanced merger of two galaxies. Furthermore, photometric and geometrical arguments indicate that the progenitors of this ultraluminous infrared system were two low-luminosity $\left(<L^{*}\right)$ spirals that could have suffered a retrograde encounter.
\end{abstract}

Subject headings: galaxies: active — galaxies: individual (IRAS 15206+3342) — galaxies: interactions — galaxies: nuclei — galaxies: starburst

\section{INTRODUCTION}

Ultraluminous infrared galaxies (ULIRGs), with bolometric luminosities $L_{\mathrm{bol}} \approx L_{\mathrm{IR}} \geq 10^{12} L_{\odot}$, are the most luminous objects in the local universe. ULIRGs show signs of strong interactions and mergers (Sanders et al. 1988a, 1988b; Melnik \& Mirabel 1990; Clements et al. 1996), and they have large amounts of gas and dust that significantly obscure their ionizing sources (Sanders \& Mirabel 1996). An evolutionary sequence has been suggested according to which "warm" (i.e., $\left.f_{25} / f_{60}>0.2\right)$ ULIRGs represent the transition phase between "cool" (i.e., $f_{25} / f_{60}<0.2$ ) ULIRGs and optical quasars (QSOs; Sanders et al. 1998a).

Much of the recent work done on ULIRGs is based on high spatial resolution imaging at different spectral regimes (e.g., Surace et al. 1998; Surace \& Sanders 1999, 2000; Surace, Sanders, \& Evans 2000; Borne et al. 2000; Scoville et al. 2000; Farrah et al. 2001; Bushouse et al. 2002; Colina et al. 2001), as well as on optical and infrared spectroscopy of the nuclear regions (Veilleux, Sanders, \& Kim 1997, 1999b; Veilleux, Kim, \& Sanders 1999a; Kim, Veilleux, \& Sanders 1998; Lutz et al. 1998). Some of these studies (e.g., Surace et

\footnotetext{
${ }^{1}$ Based on observations with the William Herschel Telescope operated on the island of La Palma by the Isaac Newton Group (ING) in the Spanish Observatorio del Roque de los Muchachos of the Instituto de Astrofísica de Canarias. Based also on observations with the NASA/ESA Hubble Space Telescope, obtained at the Space Telescope Science Institute, which is operated by the Association of Universities for Research in Astronomy, Inc., under NASA contract NAS5-26555.

${ }^{2}$ Space Telescope Science Institute, 3700 San Martin Drive, Baltimore, MD 21218; arribas@stsci.edu. Affiliated with the Research and Science Department of ESA.

${ }^{3}$ On leave from the Instituto de Astrofísica de Canarias, Consejo Superior de Investigaciones Científicas (CSIC).

${ }^{4}$ Instituto de Estructura de la Materia, Consejo Superior de Investigaciones Científicas (CSIC), Serrano 121, 28006 Madrid, Spain; colina@, isis.iem.csic.es.
}

al. 1998; Veilleux et al. 1999a) show that certain key morphological features (e.g., presence of single/double nuclei, clustering in the tidal tails, etc.) as well as the nuclear ionization properties, seem to support the evolutionary sequence proposed by Sanders et al. (1988a). However, other studies argue that ULIRGs are also generated in multiple mergers (Borne et al. 2000) or in mergers involving low massive galaxies (Bushouse et al. 2002; Colina et al. 2001). Consequently, the ULIRG evolutionary sequence toward the formation of a massive, bright quasar is not clear. Details on the processes involved remain very uncertain, despite the fact that in recent years important progress has been made in order to characterize the merger evolution on the basis of theoretical models (see Mihos \& Hernquist 1996 and references therein). These studies show that gas dynamics and star formation in mergers are closely related. They also indicate that the internal structure of the merging galaxies, rather than the orbital geometry, is a key factor in producing ULIRGs (see Mihos \& Hernquist 1996).

From an observational point of view, detailed studies of the complex kinematic and ionization structures of these objects demand the analysis of their spectral properties in two dimensions. However, only a very limited number of this type of studies exists so far (e.g., Mihos \& Bothun 1998; Wilman, Crawford, \& Abraham 1999). A program aimed at studying a representative sample of ULIRGs using integral field spectroscopy (IFS) was started a few years ago using the INTEGRAL system (Arribas et al. 1998). Some of the main results obtained thus far for other ULIRGs such as Mrk 273, IRAS 12112+0305, IRAS 08572+3915, and Arp 220 can be found elsewhere (e.g., Colina, Arribas, \& Borne 1999; Colina et al. 2000; Arribas, Colina, \& Borne 2000; Arribas, Colina, \& Clements 2001).

In this paper we present INTEGRAL Spectroscopy of IRAS $15206+3342$. The observed characteristics make of IRAS $15206+3342$ a peculiar ULIRG for which some of its 
basic properties have not yet been well established. A Seyfert 2 nucleus was reported by Sanders et al. (1988b) and Surace \& Sanders (2000), while Kim et al. (1998) identified the galaxy spectrum as $\mathrm{H}$ II-like. The near-infrared search for hidden broad-line regions (BLRs) by Veilleux et al. (1997) indicates no signs of a BLR in this object, and therefore no clear evidence for a Seyfert 1 nucleus. IRAS $15206+3342$ has the largest $\mathrm{H} \alpha$ and $\mathrm{Pa} \alpha$ equivalent widths (397 and $545 \AA$, respectively) of all ULIRGs observed spectroscopically in the optical (Veilleux et al. 1999a) and nearinfrared (Veilleux et al. 1997). The morphology of IRAS $15206+3342$ is distorted in the outer regions, the presence of a second nucleus is unclear, and inconclusive reports about the existence of tidal tails exist (Surace et al. 1998; Surace \& Sanders 2000). Optical Hubble Space Telescope (HST) WFPC2 (Surace et al. 1998) and high-resolution infrared imaging (Surace \& Sanders 1999) have shown the presence in the innermost regions of an arc-shaped chain of knots, some of which are extremely luminous $\left(M_{B}^{\prime} \sim-18\right)$. Finally, the far-infrared colors of IRAS $15206+3342$ (i.e., $\left.f_{25} / f_{60}=0.2\right)$ are on the borderline of "cold" and "warm" ULIRGs.

The new data presented here will be used to analyze the kinematics of the warm gas (two-dimensional velocity field and velocity dispersion map), as well as the stellar and ionized gas structure. The new results are discussed within the framework of the current merging models, constraining the phase of the merger and the type of progenitor galaxies involved in the merger. At the assumed distance of $540 \mathrm{Mpc}$ for IRAS $15206+3342,1^{\prime \prime}$ corresponds to a linear size of 2.6 $\mathrm{kpc}$ and throughout the paper a Hubble constant of $70 \mathrm{~km}$ $\mathrm{s}^{-1} \mathrm{Mpc}^{-1}$.

\section{OBSERVATIONS AND DATA REDUCTION}

Integral field spectroscopy of IRAS $15206+3342$ was obtained with the INTEGRAL system (Arribas et al. 1998) and WYFFOS spectrograph (Bingham et al. 1994) on the $4.2 \mathrm{~m}$ William Herschel Telescope during 1999 April 3. The bundle of fibers SB2 (standard bundle number 2), consisting of 219 fibers, each 0.9 in diameter, was used. The bundle of fibers is arranged such that 189 fibers cover a rectangular area of 16 ". $5 \times 12^{\prime \prime} .3(43 \times 32 \mathrm{kpc}$ at the distance of IRAS $15206+3342)$ centered on the galaxy, while 30 additional fibers, arranged in a ring of $90^{\prime \prime}$ in diameter concentric with the rectangle, measure simultaneously the sky background (see Arribas \& Mediavilla 2000 for further details on integral field fiber spectroscopy). The spectra were taken using a 600 line $\mathrm{mm}^{-1}$ grating with an effective resolution of $4.8 \AA$ and covering the 5000-7900 A spectral range. The total integration time was $7200 \mathrm{~s}$, split into four separate integrations of $1800 \mathrm{~s}$ each, with seeing of about 1 !" 1 .

Data reduction followed the standard procedures applied to spectra obtained with two-dimensional fiber spectrographs (Arribas et al. 1997 and references therein), which basically consist of two main steps: (1) basic reduction of the spectra (i.e., bias, flat-fielding, wavelength calibration, etc.) and (2) generation of maps of spectral features (e.g., line intensity, velocity fields, etc.) from the spectra. The basic reduction was performed inside the IRAF environment and applying standard two-dimensional spectroscopy calibration routines. Maps of spectral features were generated using a Renka \& Cline two-dimensional interpolation method to transform a file with the actual position of the fibers and the spectral feature (i.e., line emission, continuum within a window, radial velocity, line width, etc.) into a regularly spaced rectangular grid. In this way, images that can be treated with standard astronomical software were built up. As an example, the two-dimensional distribution of the reduced spectra in the range of the redshifted $\mathrm{H} \alpha+[\mathrm{N}$ II] lines (i.e., 7310-7480 $\AA$ ) are presented in Figure 1. Other emission lines such as $\mathrm{H} \beta$, [O III] $\lambda 5007$, [O $\mathrm{I}] \lambda 6300$, and [S II] $\lambda \lambda 6716,6731$ have also been analyzed.

For each spectrum, the radial velocities and velocity dispersions were measured by adjusting single Gaussian functions to the observed emission line profiles using the DIPSO package (Howarth \& Murray 1988). Kinematic maps for the $\mathrm{H} \beta,[\mathrm{O}$ III $] \lambda 5007$, and $\mathrm{H} \alpha-[\mathrm{N} \mathrm{II}]$ emission lines were obtained. However, since the results from these different lines agree within the uncertainties, the maps used in the discussion correspond to the $\mathrm{H} \alpha$ and [N II] lines as they have a higher signal-to-noise ratio and cover a wider field of view. The statistical uncertainties associated with the individual velocities are very small as indicated by the mean difference of $-1 \pm 15 \mathrm{~km} \mathrm{~s}^{-1}$ between the velocities derived using the [O III] and the $\mathrm{H} \alpha-[\mathrm{N}$ II] lines. The velocity dispersion values presented in this paper are corrected from instrumental profile and redshift.

To investigate certain morphological features of the stellar component, archival $H S T /$ WFPC2 images of IRAS $15206+3342$ obtained in the HST B and $I$ bands (F439W and F814W filters, respectively) have been used. These archival images, first analyzed by Surace et al. (1998), were recalibrated using the on-the-fly calibration software and the best reference calibration files available. The calibrated images were filtered with a long bandpass median filter $(15 \times 15$ pixels $)$ to enhance the lowest surface brightness regions. Taking into account the poorer spatial resolution, and the slightly different effective wavelength, the INTEGRAL red continuum image taken with a narrow 7480 $7510 \AA$ window has a good morphological resemblance with the WFPC2 I-band image (Fig. 2). Therefore, the absolute positioning of IRAS $15206+3342$ in the INTEGRAL maps is known to within 0 ". 3 of the HST coordinates.

\section{RESULTS AND DISCUSSION}

\subsection{Stellar and Ionized Gas Structures: Morphological Evidence for a Tidal Tail}

The structure of the warm ionized gas and internal dust extinction is traced here by the $\mathrm{H} \alpha,[\mathrm{O}$ III] $\lambda 5007$, [S II] $\lambda \lambda 6716,6731$ emission lines and the $\mathrm{H} \alpha / \mathrm{H} \beta$ line ratio maps, respectively (Fig. 2).

The warm ionized gas structure shows a high surface brightness region that is elongated along the east-west direction, consistent with the 1"1-convolved light distribution of the arc-shaped chain of bright knots detected in the main body of the galaxy (Surace et al. 1998). The ionized gas distribution peaks in the eastern region of the galaxy where the brightest optical and near-infrared-emitting knot, identified as the nucleus of the galaxy, is located (Surace et al. 1998; Surace \& Sanders 1999). Moreover, since the highest extinction is found in this region of the galaxy $(\mathrm{H} \alpha / \mathrm{H} \beta \sim 6.8$ equivalent to $A_{v}=2.2 \mathrm{mag}$; Fig. 2), the true (i.e., dereddened) ionized gas distribution is even more concentrated and therefore associated with the nucleus of the galaxy. 


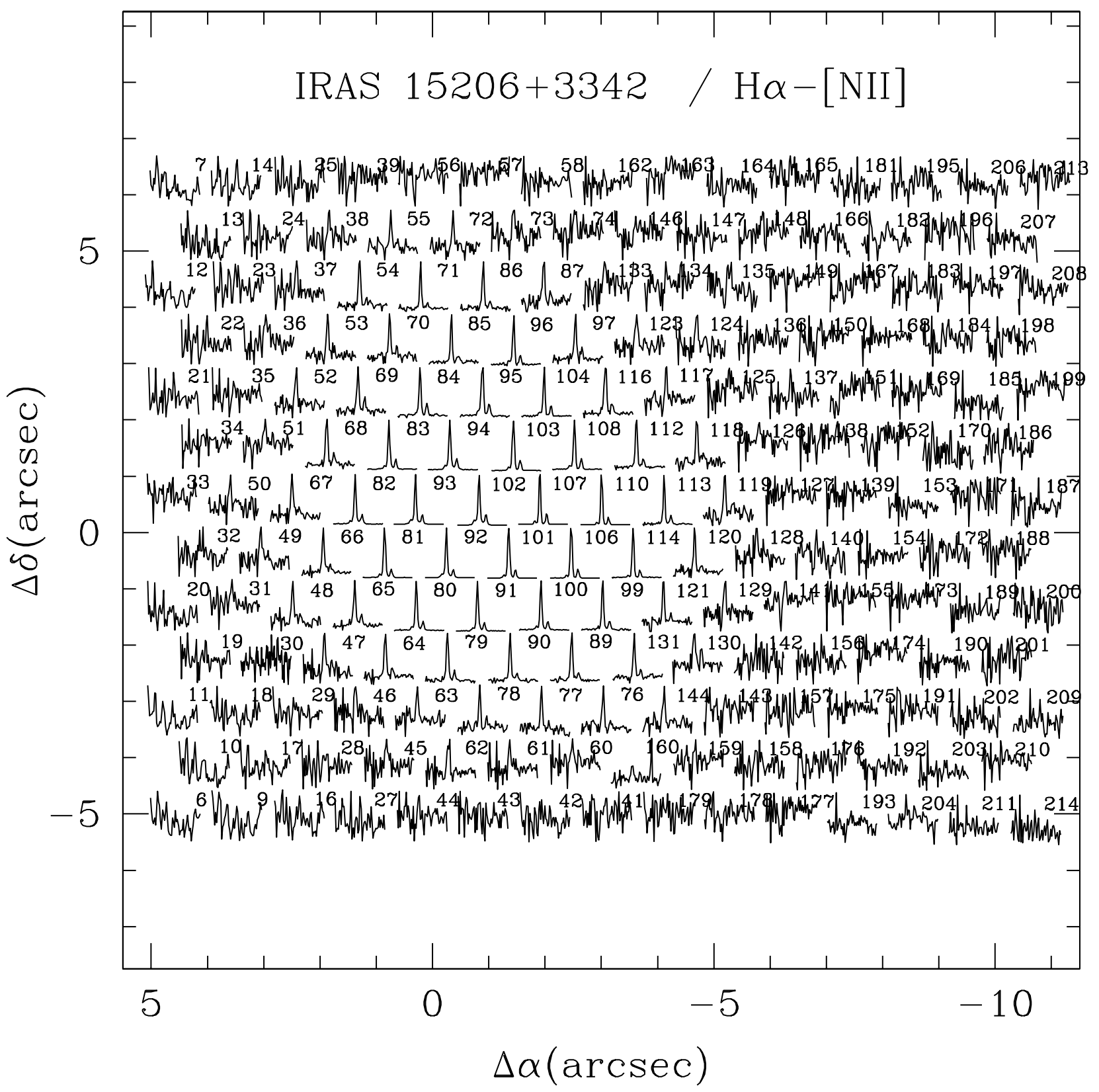

FIG. 1.- Spatial distribution of the spectra in the spectral range 7310-7480 A corresponding to the redshifted H $\alpha+[\mathrm{N}$ II] emission line complex. Numbers indicate the fiber/spectrum position at the slit/detector. These spectra are scaled between their lowest and highest values in the represented spectral range.

In addition to the main structure, the ionized gas distribution shows the presence of a low surface brightness plume bent toward the northeast of the nucleus (Fig. 2). This plume of gas is identified as a partially broken tidal tail that we also detected in the filtered I-band WFPC2 image (Fig. 3).

The tail has a linear size of $\sim 13 \mathrm{kpc}$, and the inner regions are characterized by a surface brightness of $m_{I} \sim 21.5-22.0$ mag $\operatorname{arcsec}^{-2}$ and a color $B-I$ of about 0.5 . The outer part of the tail possibly extends to a resolved stellar system located at position $\left(+7{ }^{\prime \prime} 7,+3\right.$ "' 8$)$, i.e., at a projected distance of about $20 \mathrm{kpc}$ from the nucleus, of 0.77 in size $(2 \mathrm{kpc})$, and with an apparent integrated $I$ magnitude $m_{I} \sim 23.8$ corresponding to $M_{I} \sim-14.8$, assuming it is at the same redshift as IRAS $15206+3342$. This structure is not detected in the
$B$-band image, implying it has a $B-I>0.7$. The overall bent structure of the gaseous and stellar tail is such that it delineates a spiral arm connecting the resolved extranuclear stellar remnant with the innermost arc-shaped chain of starforming knots close to the nucleus. The $\mathrm{H} \alpha / \mathrm{H} \beta$ extinction map also shows the same spiral-like structure where internal extinction increases inward along the tidal tail from $A_{V} \sim 1.4 \mathrm{mag}$ in the external region of the tail up to $A_{V} \sim 2.2 \mathrm{mag}$ in the nucleus, while the ionized regions are nearly free of extinction (i.e., $\mathrm{H} \alpha / \mathrm{H} \beta \sim 3$ ) elsewhere. Finally, no $\mathrm{H}$ in regions or dwarf star-forming galaxies along the most external regions of the tidal tail-similar to those found in some double-nuclei ULIRGs (e.g., IRAS 12112+0305: Colina et al. 2000; IRAS 08572+3915: Arribas et al. 2000) - have been detected. However, the low surface 

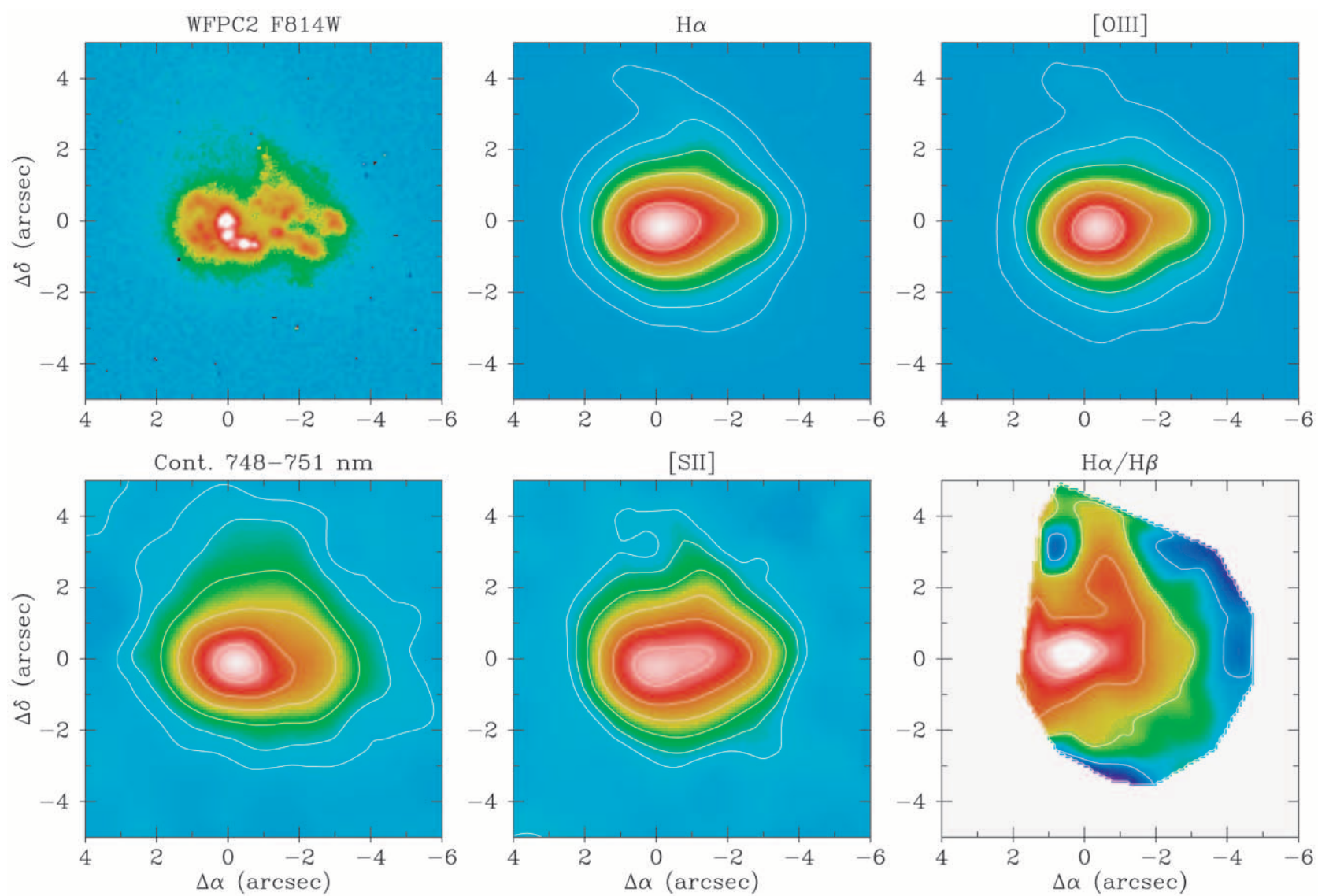

FIG. 2.-INTEGRAL images of the ionized gas and stellar light distribution in the central regions of IRAS $15206+3342$, as traced by different emission lines and by a continuum window (7480-7510 A). The archival high-resolution HST I-band image (Surace et al. 1998) is also shown for comparison. The presence of the tidal tail in the $\mathrm{H} \alpha$ and $[\mathrm{O}$ III] line maps is clearly visible. The differential internal extinction map traced by the $\mathrm{H} \alpha / \mathrm{H} \beta$ ratio is also shown. The emission lines maps are continuum-subtracted. They have the following isocontour values (in arbitrary units). $\mathrm{H} \alpha: 0.9,2.4,6.2,16,40,100 ;[\mathrm{O}$ III]: 0.6, 2.1, 5.9, 15, 39, 100; Cont: $0.2,3.4,7.2,16,40,100 ;[\mathrm{S}$ II] $2.8,4.3,8,17,41,100 ; \mathrm{H} \alpha / \mathrm{H} \beta: 2.5,3.5,4.5,5.5$. North is at the top, and east is to the left.

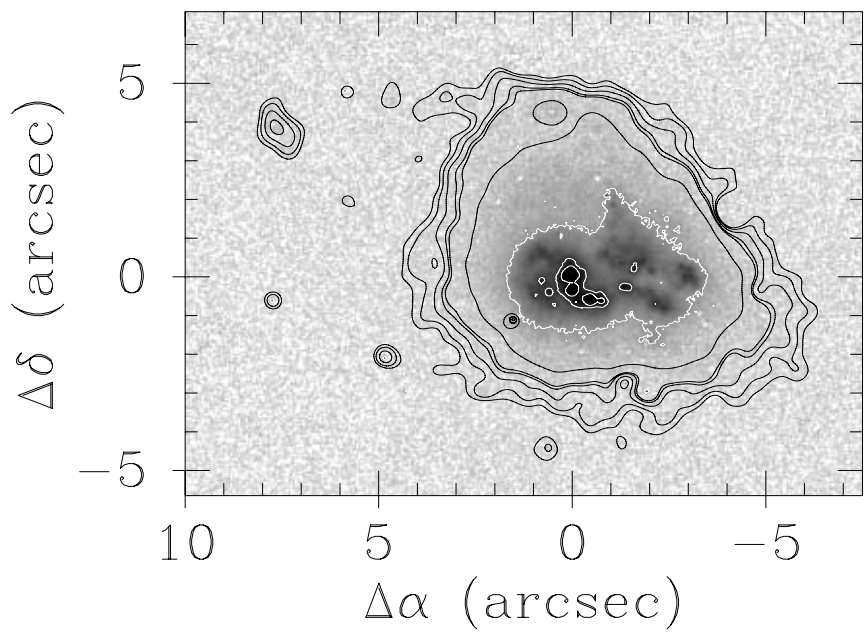

FIG. 3.- HST/WFPC2 I-band image. White isolines correspond to the original high spatial resolution $H S T$ WFPC2 image. The arc-shaped chain of knots reported by Surace et al. (1998) is clearly visible. Black isolines correspond to the filtered WFPC2 I-band image (see $\S 2$ ). These isoline levels have been selected to enhance the low surface brightness stellar component of the tidal tail and the stellar remnant at $(7 "$ ' 7,3 ,"8). brightness of the tail and its lack of external star-forming knots is characteristic of single-nucleus ULIRGs such as IRAS 20551-4250 (Mihos \& Bothun 1998).

\subsection{Two-dimensional Kinematics of the Ionized Gas: Evidence for Inflow along the Tidal Tail}

The ionized gas velocity field traced by the $\mathrm{H} \alpha-[\mathrm{N}$ II] lines shows a rather complex structure (Fig. 4). The largest velocity gradient is not found across the nucleus but across the region at $2^{\prime \prime}(5 \mathrm{kpc})$ northwest of the nucleus where the tidal tail connects with the main body of the galaxy. The maximum peak-to-peak velocity difference corresponds to $\delta V=250 \mathrm{~km} \mathrm{~s}^{-1}$ along position angle (P.A.) $\sim 30^{\circ}$. On the other hand, the velocity dispersion map (Fig. 4) shows relatively large velocity dispersions $\left(\sigma \sim 120 \mathrm{~km} \mathrm{~s}^{-1}\right)$ over the entire field, with the exception of two regions that are in good positional agreement with the northern part of the tidal tail and the western part of the galaxy where low values of $\sigma \sim 50 \mathrm{~km} \mathrm{~s}^{-1}$ are found.

The velocity field and the velocity dispersion maps are rather symmetric around the region located $2^{\prime \prime}$ northwest but can hardly be interpreted as rotation, since most of the mass is located around the bright $H$-band nucleus, and there is no evidence for a large mass concentration in this region (see $\S 3.1$ ). The observed gas velocity pattern clearly corre- 

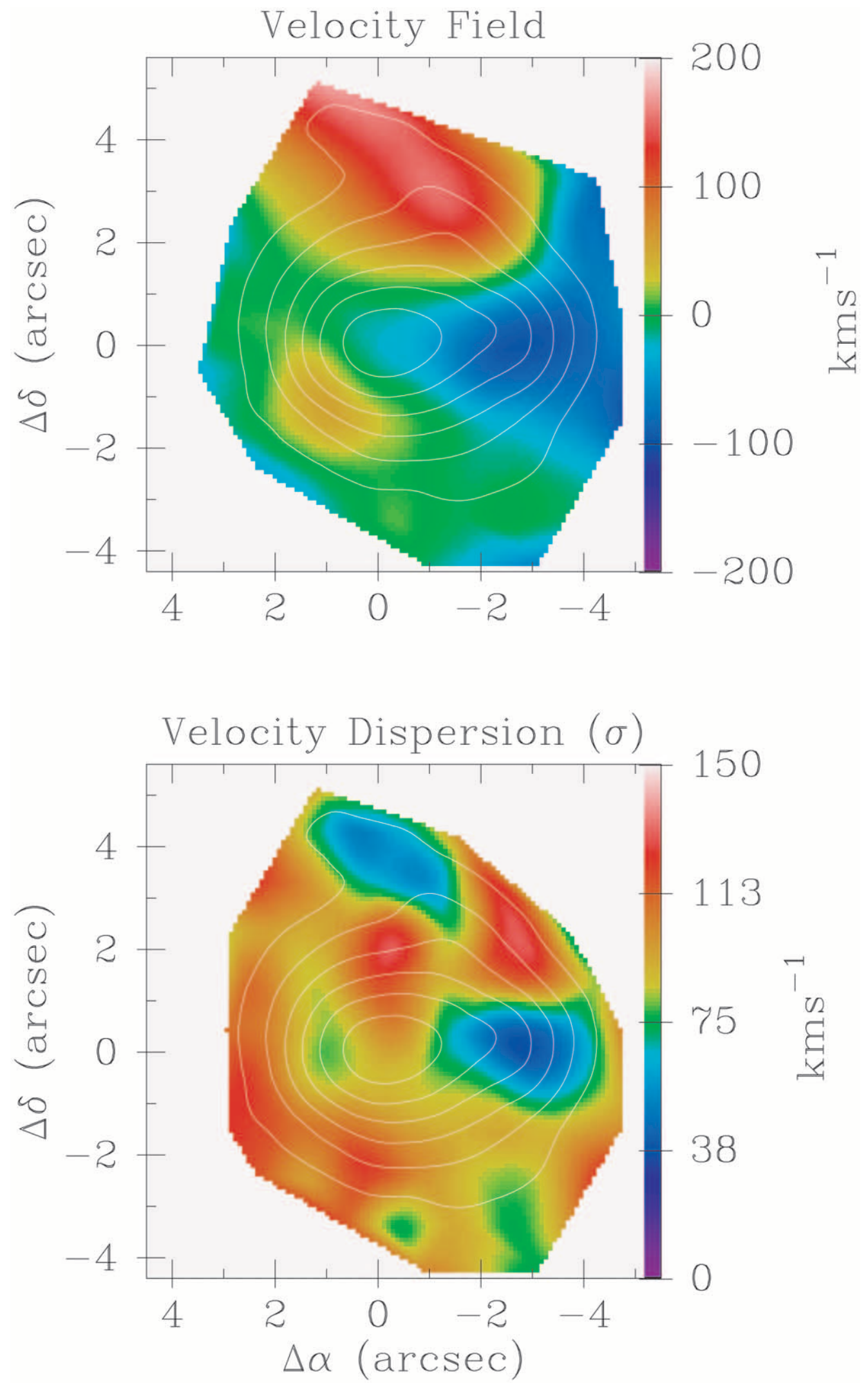

FIG. 4.-Velocity field and dispersion maps inferred from the $\mathrm{H} \alpha+[\mathrm{N}$ II $]$ lines. Isolines correspond to the $\mathrm{H} \alpha$ intensity map. Note that the large velocity gradient and small velocity dispersions are found across a region centered at $2^{\prime \prime}$ northwest of the nucleus (P.A. $\sim 30^{\circ}$ ), following the tidal tail traced by the $\mathrm{H} \alpha$ emission. 
lates in position with the tidal tail, indicating that the observed velocities are associated with flows of gas along the tail. In principle, both outflows or inflows of gas should be considered. However, the presence of an outflow is difficult to sustain since there is no evidence for the existence of an energetic ionizing source (young massive stars or active galactic nuclei [AGNs]) at this location that could generate a powerful bipolar outflow with velocity gradients of $\sim 50$ $\mathrm{km} \mathrm{s}^{-1} \mathrm{kpc}^{-1}$ along P.A. $30^{\circ}$.

Alternatively, the velocity field along the tidal tail is better explained as a result of gas inflow. Under this hypothesis the tidal tail should be strongly bent (see Fig. 5 for an illustrative sketch), in order to explain the observed change in velocity of $250 \mathrm{~km} \mathrm{~s}^{-1}$ within a region of less than $5 \mathrm{kpc}$ (Fig. 4). This interpretation is also supported by the velocity dispersion map. As expected, the lowest velocity dispersions $\left(\sigma \sim 50 \mathrm{~km} \mathrm{~s}^{-1}\right)$ are found along the tidal tail, except in the region where velocities change from redshifted to blueshifted values (Fig. 4).

The light distribution within the inner region of $\sim 1 " 5$ (3.9 $\mathrm{kpc}$ ) is well centered with the optical nucleus and has a circular/elliptical shape (Fig. 4). This suggests that the observed velocity field in the $4 \mathrm{kpc}$ region around the nucleus of IRAS $15206+3342$ could be represented by a low-inclination rotating disk, whose western part shows approaching velocities, similar to the velocities measured at this position for the gas in the tail. This result supports a kinematical connection of the tidal tail with the nuclear regions. Finally, the velocity field does not show any kinematical evidence for the presence of a second nucleus at a distance of $4 \mathrm{kpc}$, or larger, from the main nucleus of the galaxy, and with a velocity of more than about $50 \mathrm{~km} \mathrm{~s}^{-1}$ relative to that of the nucleus.

In summary, the kinematic structure along the tidal tail is consistent with gas inflows toward the innermost regions of IRAS $15206+3342$. The existence of such inflows supports the idea that the tail is channeling gas from the external regions several kpc away into the nuclear regions where a massive starburst, distributed along an arc-shaped chain of star-forming knots, is taking place. As we will see in $\S 3.4$, the presence of strong inflows and associated star formation are expected during the final coalescence phase of mergers (Mihos \& Hernquist 1996).

\subsection{Warm Line-emitting Gas Nebula: Evidence for a Starburst-dominated Ionizing Source}

The standard gas excitation diagnostic diagrams are presented in Figure 6. Each point in these diagrams corresponds to an individual spectrum (i.e., a location). A clear dichotomy exists between the excitation properties of the nucleus (Fig. 6, filled squares) and circumnuclear regions (Fig. 6, crosses). The extended circumnuclear regions, including the tidal tail and the region $2^{\prime \prime}$ west of the nucleus, favor a LINER-like ionization according to the $\log ([\mathrm{O} \mathrm{III}] / \mathrm{H} \beta)$ versus $\log \left[\mathrm{O}_{\mathrm{I}}\right] / \mathrm{H} \alpha$ and, to a lesser degree, the $\log ([\mathrm{O} \mathrm{III}] / \mathrm{H} \beta)$ versus $\log ([\mathrm{S} \mathrm{II}] / \mathrm{H} \alpha)$ diagrams. As in other luminous infrared galaxies (e.g., Arp 220; Arribas et al. 2001), the detection of a LINER spectrum in extended kiloparsec-size regions is likely due to shock excitation induced by the combined effect of massive stellar winds (Heckman, Armus, \& Miley1990).

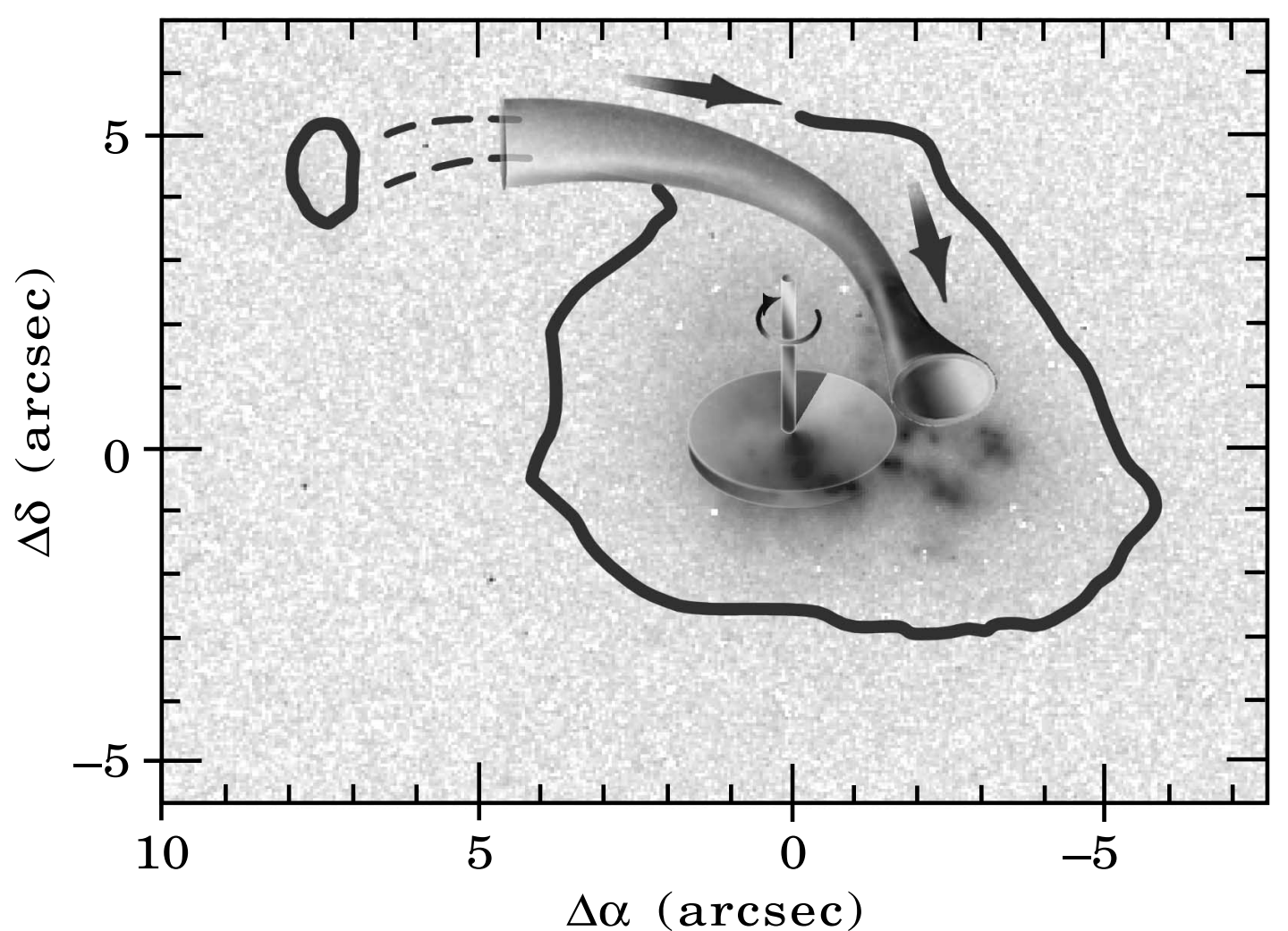

FIG. 5.- Sketch of the proposed three-dimensional structure in IRAS $15206+3342$. A bent tidal tail of about $15-20$ kpc in projected length connects the inner arc-shaped chain of star-forming knots with a remnant stellar system located at about $20 \mathrm{kpc}$ from the nucleus. The gas in the tidal tail is flowing inward the inner disk. 


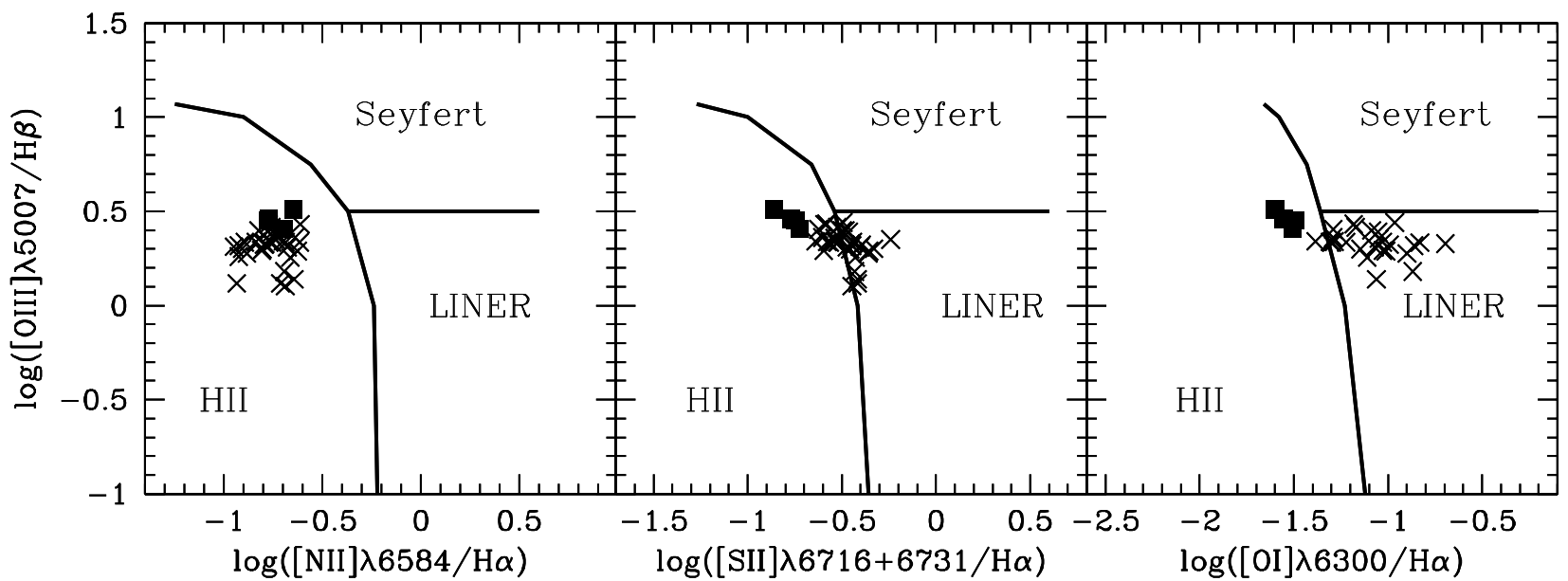

Fig. 6.-Excitation diagnostic diagrams are presented for the nucleus ( filled squares) and for the extranuclear regions, including the tidal tail (crosses). Typical errors are on the order of the symbol size.

The nucleus has an unequivocally $\mathrm{H}$ II-type ionization in the three available diagrams. Note that there are many examples of $\mathrm{H}$ II-like ionization in the nuclear regions of ULIRGs, although the classification may depend on the line ratios used (see, for instance, Veilleux et al. 1999b, Table 5). Our values for the nucleus $[0.46,-0.77,-0.77,-1.55$ for $\log \left(\left[\mathrm{O}_{\mathrm{III}}\right] / \mathrm{H} \beta\right), \quad \log \left(\left[\mathrm{N}_{\mathrm{II}}\right] / \mathrm{H} \alpha\right), \quad \log \left(\left[\mathrm{S}_{\mathrm{II}}\right] / \mathrm{H} \alpha\right), \quad$ and $\log \left(\left[\mathrm{O}_{\mathrm{I}}\right] / \mathrm{H} \alpha\right)$, respectively] agree well with those of Kim et al. (1998), who also indicated an H II-like ionization and disagree with previous claims of a Seyfert 2 nucleus (Sanders et al. 1988b). However, there are arguments suggesting that the nucleus of IRAS $15206+3342$ is a luminous AGN. The equivalent width of the nuclear $\mathrm{H} \alpha$ line is extremely large, with a value $\mathrm{EW}(\mathrm{H} \alpha)=410 \pm 10 \AA$ in good agreement with a previous measurement of 397 A by Veilleux et al. (1999a). An extremely large equivalent width of $545 \AA$ has also been measured by the same authors (Veilleux et al. 1997) for the $\operatorname{Pa} \alpha$ emission line. These equivalent widths are the largest of all ULIRGs for which optical and near-infrared spectroscopy is available and place the nucleus of IRAS $15206+3342$ among the class of Seyfert 1 ULIRGs. Moreover, the measured nuclear $\mathrm{H} \alpha$ equivalent width is a factor of 4.9, 4.7, and 8.2 larger, respectively, than the average value for ULIRGs classified as Seyfert 2 (84 ̊), H II ( $88 \AA$ ), and LINER (50 A) obtained by Veilleux et al. (1999a). Also, the observed nuclear $B$-band luminosity $\left(m_{B}=19.3\right.$; Surace et al. 1998), dereddened assuming the blue continuum has the same extinction as the emission lines (i.e., $A_{V}=2.2$; see $\S 3.1$ ), corresponds to an absolute magnitude $M_{B}=-22.3$, typical of blue-selected, low-luminosity, low-redshift QSOs.

Can these properties trace unequivocally the presence of a powerful Seyfert 1/QSO nucleus in IRAS $15206+3342$ ? At a distance of $540 \mathrm{Mpc}$, the region identified as the nucleus in our ground-based optical data has a linear size of about $3 \mathrm{kpc}$ (i.e., 1".2 diameter) and corresponds to the region where the arc-shaped chain of massive star-forming knots have been previously detected (Surace et al. 1998; Surace \& Sanders 1999). Extreme large $\mathrm{H} \alpha$ equivalent widths [i.e., $\mathrm{EW}(\mathrm{H} \alpha)>250 \AA$ ] have already been detected in the extranuclear regions of some ULIRGs such as IRAS $12112+0305$ (Colina et al. 2000) and IRAS 08572+3915 (Arribas et al. 2000). These regions are located along the external parts of tidal tails and have been identified as tidal- induced star-forming knots where young ( $\sim 5 \mathrm{Myr})$ clusters with masses of about $10^{6}-10^{7} M_{\odot}$ are the ionizing sources. Although in the case of IRAS $15206+3342$ the clusters are found near the nucleus, the morphological and kinematic connection of this region with the tidal tail (see $\S 3.2$ ) suggests that the arc-shaped chain of clusters could be part of the innermost tail seen in projection against the nucleus.

Finally, if the $\mathrm{H} \alpha$-emitting gas were entirely ionized by the massive stars generated in the nuclear star-forming knots, the large $\mathrm{H} \alpha$ equivalent width and the dereddened $\mathrm{H} \alpha$ luminosity $\left[\log L(\mathrm{H} \alpha)=43.2 \mathrm{ergs} \mathrm{s}^{-1}\right]$ would indicate the presence of a powerful $50 \mathrm{Myr}$ continuous starburst forming stars at a rate of about $150 M_{\odot} \mathrm{yr}^{-1}$, for a Salpeter initial mass function and stars with masses in the $0.1-100$ $M_{\odot}$ range, according to spectral synthesis models (Leitherer et al. 1999). The absolute blue magnitude of such a continuous burst would be $M_{B}=-22.7$, in agreement with the measured dereddened magnitude of -22.3 . The bolometric luminosity emitted by such a continuous starburst would be $\log L_{\text {burst }}=11.9 L_{\odot}$, or $50 \%$ of the integrated IR luminosity $\left(\log L_{\mathrm{IR}}=12.18 L_{\odot}\right)$.

\subsection{Inferences on the Merger}

In previous sections we have presented evidence supporting the conclusion that IRAS $15206+3342$ is the result of a merging process, i.e., the presence of a tidal tail, the likely existence of a remnant stellar system at about $20 \mathrm{kpc}$ from the nucleus, large velocity gradients in extranuclear regions, and disturbed ambient gas over regions of 20-30 kpc in size. Furthermore, we have found that the stellar and ionized gas distributions of IRAS $15206+3342$ are consistent with the presence of a single nucleus, the existence of an inflow of gas along the tidal tail (which is transferring mass to the inner regions), the lack of star-forming clusters along the outer parts of the tidal tail, and the presence of a powerful nuclear starburst that substantially contributes to the energy output. These results can be used to infer the phase of the merger, the general properties of the progenitor galaxies, and the type of encounter.

Recent theoretical work on modeling the evolution of mergers indicates that the internal structure of the merging galaxies, rather than orbital geometry, may be the impor- 
tant factor in producing ultraluminous infrared galaxies (Mihos \& Hernquist 1996). These models use a hybrid $N$ body/hydrodynamics code (Hernquist \& Katz 1989) modified to include star formation in the gas (Mihos \& Hernquist 1994). In the case of mergers of disk galaxies of comparable mass they found that the galaxies experience strong gaseous inflows and moderate to intense starburst activity. According to these models, galaxies without bulges would generate mild, long-lived starbursts ( $200 \mathrm{Myr})$ well before the final coalescence of the galaxies. However, strong inflows of gas and massive short-lived (i.e., 50 Myr old) starbursts forming stars at a high rate (i.e., SFR $\sim 150 M_{\odot} \mathrm{yr}^{-1}$ or more) as the one detected in the nucleus of IRAS $15206+3342$, are expected at the center of a merging system during the final coalescence phase of two disk galaxies with bulges (Mihos \& Hernquist 1996). The lack of kinematic evidence for a second nucleus at distances larger than a few kiloparsecs from the identified nucleus, and the absence of young, recently formed stellar clusters, or dwarf star-forming galaxies, along the external part of the tidal tail further support the conclusion that this galaxy is indeed an advanced merger in the final process of coalescing. In addition, the integrated $H$-band luminosity of the entire system is $M_{\mathrm{H}} \sim-24.5$ $\left(m_{\mathrm{H}}=14.14\right.$; Surace \& Sanders 1999), which corresponds to a $1.3 L^{*}$ galaxy and therefore indicates that the progenitor galaxies that are now coalescing were two low-luminosity (i.e., $<L^{*}$ ) spirals, as in many other ULIRGs (Colina et al. 2001). Finally, opposite to the long tidal tails generated during prograde encounters, the short length and geometry of the tidal tail in IRAS $15206+3342$ suggest that the encounter could have been retrograde (Mihos \& Hernquist 1996) if massive halos were not present in the progenitor galaxies (Dubinski, Mihos, \& Hernquist 1999).

In summary, the kinematical, morphological, and photometric evidence presented here supports the idea that we are witnessing in IRAS $15206+3342$ the final coalescence phase of a system composed of two low-luminosity spirals, each consisting of a disk + bulge internal structure, that have been involved in a retrograde encounter.

\section{SUMMARY}

Integral field optical fiber spectroscopy with the INTEGRAL system, together with archival HST/WFPC2 images, have been used to investigate the region of $25 \mathrm{kpc}$ surrounding the nucleus of the ultraluminous infrared galaxy IRAS $15206+3342$. The main results are the following:

1. The stellar and ionized gas distributions reveal the presence of a low surface brightness tidal tail of about 20 $\mathrm{kpc}$ in size that connects an extranuclear remnant stellar system with the nuclear arc-shaped star-forming knots detected previously by Surace \& Sanders (1999).

2. The velocity field and the velocity dispersion maps indicate the presence of gas inflow along a bent tidal tail that likely transports gas into the innermost regions and therefore fuels the ongoing nuclear starburst.

3. There is no kinematical evidence for a second nucleus. However, a second nucleus could still exist at a distance of less than $\sim 4 \mathrm{kpc}$ from the identified nucleus and/or with a velocity of less than $\sim 50 \mathrm{~km} \mathrm{~s}^{-1}$ relative to the nucleus.

4. The ionization conditions of the nucleus are those of a $\mathrm{H}$ II-like nebula, and there are not traces of Seyfert emission. However, shock-induced LINER ionization is present in the circumnuclear ionized regions, including the $15 \mathrm{kpc}$ tidal tail.

5. The nuclear $\mathrm{H} \alpha$ emission characterized by an extremely large equivalent width of $410 \AA$, and a luminosity of $\log L(\mathrm{H} \alpha)=43.2 \mathrm{ergs} \mathrm{s}^{-1}$, is consistent with a $50 \mathrm{Myr}$ old continuous starburst forming stars at a rate of $150 \mathrm{M}_{\odot}$ $\mathrm{yr}^{-1}$ and contributing at least $50 \%$ of the bolometric luminosity of IRAS $15206+3342$.

6. According to the hydrodynamic models of Mihos \& Hernquist (1996), the observed kinematic and photometric properties of IRAS $15206+3342$ are consistent with this system being an advanced merger in the final coalescence phase of two subluminous $\left(<L^{*}\right)$ disk galaxies with a bulge that have experienced a retrograde encounter.

We thank all the staff at the Spanish Observatorio del Roque de los Muchachos of the Instituto de Astrofísica de Canarias for their support. We also thank Luis Cuesta for his help using GRAFICOS. Support for this work was provided by CICYT (Comisión Interministerial de Ciencia y Tecnología) through grants PB98-0340-C01 and PB980340-C02.
Arribas, S., Colina, L., \& Borne, K. D. 2000, ApJ, 545, 228

Arribas, S., Colina, L., \& Clements, D. 2001, ApJ, 560, 160

Arribas, S., \& Mediavilla, E. 2000, in ASP Conf. Ser. 195, Imaging the Universe in Three Dimensions: Astrophysics with Advanced Multiwavelength Imaging Devices, ed. W. van Breugel \& J. Bland-Hawthorn (San Francisco: ASP), 295

Arribas, S., Mediavilla, E., García-Lorenzo, B., \& del Burgo, C. 1997, ApJ, 490, 227

Arribas, S., et al. 1998, Proc. SPIE, 3355, 821

Bingham, R. G., Gellatly, D. W., Jenkins, C. R., \& Worswick, S. P. 1994, Proc. SPIE, 2198, 56

Borne, K. D., Bushouse, H., Lucas, R. A., \& Colina, L. 2000, ApJ, 529, L77

Bushouse, H., et al. 2002, ApJS, 138, 1

Clements, D. L., Sutherland, W. J., McMahon, R. G., \& Saunders, W. 1996, MNRAS, 279, 477

Colina, L., Arribas, S., \& Borne, K. D. 1999, ApJ, 527, L13

Colina, L., Arribas, S., Borne, K. D., \& Monreal, A. 2000, ApJ, 533, L9

Colina, L., et al. 2001, ApJ, 563, 546

Dubinski, J., Mihos, J. C., \& Hernquist, L. 1999, ApJ, 526, 607

Farrah, D., et al. 2001, MNRAS, 326, 1333

Heckman, T. M., Armus, L., \& Miley, G. K. 1990, ApJS, 74, 833

Hernquist, L., \& Katz, N. 1989, ApJS, 70, 419

Howarth, I. D., \& Murray, J. 1988, DIPSO-A Friendly Spectrum Analysis Program (Starlink User Note 50; Chilton: Rutherford Appleton Lab.)
REFERENCES

Kim, D. C., Veilleux, S., \& Sanders, D. B. 1998, ApJ, 508, 627

Leitherer, C., et al. 1999, ApJS, 123, 3

Lutz, D., Spoon, H. W. W., Rigopoulou, D., Moorwood, A. F. M., \& Genzel, R. 1998, ApJ, 505, L103

Melnick, J., \& Mirabel, I. F. 1990, A\&A, 231, L19

Mihos, J. C., \& Bothun, G. D. 1998, ApJ, 500, 619

Mihos, J. C., \& Hernquist, L. 1994, ApJ, 437, 611 1996, ApJ, 464, 641

Sanders, D. B., \& Mirabel, I. F. 1996, ARA\&A, 34, 749

Sanders, D. B., Soifer, B. T., Elias, J. H., Madore, B. F., Matthews, K., Neugebauer, G., \& Scoville, N. Z. 1988a, ApJ, 325, 74

Sanders, D. B., Soifer, B. T., Elias, J. H., Neugebauer, G., \& Matthews, K. 1988b, ApJ, 328, L35

Scoville, N. Z., et al. 2000, AJ, 119, 991

Surace, J. A., \& Sanders, D. B. 1999, ApJ, 512, 162 2000, AJ, 120, 604

Surace, J. A., Sanders, D. B., \& Evans, A. S. 2000, ApJ, 529, 170

Surace, J. A., Sanders, D. B., Vacca, W. D., Veilleux, S., \& Mazzarella, J. M. 1998, ApJ, 492, 116

Veilleux, S., Kim, D. C., \& Sanders, D. B. 1999a, ApJ, 522, 113

Veilleux, S., Sanders, D. B., \& Kim, D. C. 1997, ApJ, 484, 92

. 1999b, ApJ, 522, 139

Wilman, R. J., Crawford, C. S., \& Abraham, R. G. 1999, MNRAS, 309, 299 